\title{
Unexpected Occurrence of Caffeine in Sleep-Inducing Herbal Teas
}

\author{
Caroline Mathon ${ }^{\mathrm{ab}}$, Patrick Edder ${ }^{\mathrm{a}}$, Philippe Christen ${ }^{\star \mathrm{b}}$, and Stefan Bieri ${ }^{\mathrm{a}}$
}

\begin{abstract}
Caffeine acts as a weak psychostimulant and is known to cause trouble with sleeping. Therefore, the presence of caffeine in sleep-aid herbal teas was somewhat surprising, and confirmatory investigations were conducted to exclude any possible misidentification. The botanicals of the sedative mixtures were analysed individually by ultra performance liquid chromatography (UPLC) coupled with a time-of-flight high-resolution mass spectrometer (TOF-HRMS), and caffeine was detected in linden (Tilia spp.) extracts. The presence of caffeine was unambiguously confirmed by means of its characteristic mass spectrum acquired during direct analyses of powdered linden by thermal desorption coupled to a GC×GC-TOF-MS. Caffeine content was determined in 11 linden-based samples, with a validated UPLC-MS/MS method using two mass transitions. Concentrations were between traces and $110 \mathrm{mg} \mathrm{kg}^{-1}$ in the herbal material while those in the corresponding prepared sleep-inducing hot beverages ranged from traces to $226 \mu \mathrm{g}$ per cup.
\end{abstract}

Keywords: Caffeine $\cdot$ Herbal tea $\cdot$ Linden $\cdot$ Tilia spp. · UPLC-MS/MS

\section{Introduction}

Caffeine occurs naturally in a large variety of botanicals (e.g. coffee, tea, guarana, mate, kola nut), ${ }^{[1]}$ which are popularly used to prepare beverages all around the world. In addition caffeine is frequently added to food supplements or energy drinks. This trimethylxanthine is the most consumed drug all over the world due to its various psychostimulant properties. ${ }^{[2]}$ In cases of excessive consumption, disorientation, agitation or insomnia are frequently reported. ${ }^{[3,4]}$

During a survey of more than 100 herbal dietary supplements, caffeine was often detected in slimming supplements but also in some linden-based sleep-aid phytopreparations. ${ }^{[5]}$ Due to the antagonist properties of linden ${ }^{[6-8]}$ (Tilia spp.) and caffeine, the detection of the latter was quite unexpected in these foodstuffs. Although caffeine has been identified in linden honey, ${ }^{[9]}$ to the best of our knowledge, caffeine has never been detected in linden teas. ${ }^{[10,11]}$

This article reports on the occurrence of caffeine in linden teas using several analytical approaches. Investigations have been carried out on linden plant material by accelerated solvent extraction (ASE) and extracts were analysed using ultra performance liquid chromatography (UPLC)

\footnotetext{
${ }^{*}$ Correspondence: Dr. P. Christen ${ }^{b}$

Tel.: +41223796561

E-mail: Philippe.Christen@unige.ch

afficial Food and Veterinary Control Authority of Geneva

Quai Ernest Ansermet 22

$\mathrm{CH}-1211$ Geneva 4

School of Pharmaceutical Sciences,

EPGL, University of Geneva, University of Lausanne

Quai Ernest Ansermet 30

$\mathrm{CH}-1211$ Geneva
}

coupled to a time-of-flight (TOF) highresolution mass spectrometer (HRMS). Powdered linden materials were also directly desorbed using a thermal desorption unit (TDU), and volatile and semi-volatile compounds were detected by TOF-MS after a comprehensive two-dimensional gas chromatography $(\mathrm{GC} \times \mathrm{GC})$ separation. For compound identification, generated mass spectra were compared with those of the NIST library.

Once caffeine was unambiguously identified using orthogonal methods, it was quantified in 11 samples of linden, using a validated UPLC-MS/MS method. The LC separation was based on the method described by Eugster et al. ${ }^{[12]}$ Amounts of caffeine were measured in linden tea and powdered linden extracted by ASE with a methanol-water mixture, as suggested by Jun. ${ }^{[13]}$ Finally, to evaluate the correlation between caffeine concentrations in linden tea and brewing times, amounts were measured at several steeping times.

\section{Materials and Method}

\subsection{Chemicals and Samples}

All chemicals and HPLC grade solvents were purchased from Sigma-Aldrich ${ }^{\circledR}$ (Buchs, Switzerland). Water was purified with a Milli-Q system from Millipore (Molsheim, France).

Linden flowers (teabags and loose samples) were bought in supermarkets and pharmacies in Switzerland.

\subsection{Sample Preparations}

Boiled water (200 mL) was gently poured over a teabag (or $1 \mathrm{~g}$ of loose plant material). After $5 \mathrm{~min}$ of brewing under slight agitation at room temperature, herbal teas were centrifuged for $5 \mathrm{~min}$ at $5000 \mathrm{~g}$, at room temperature. Supernatants were filtered through a $0.2 \mu \mathrm{m}$ PTFE filter (Infochroma, Zug, Switzerland) and aliquots $(50 \mu \mathrm{L})$ were diluted four times.

Powdered linden samples were extracted using an ASE 200 (Thermo Scientific Dionex, Olten, Switzerland). Plant material (200 mg) was mixed and homogenised with quartz sand and introduced into an 11 $\mathrm{mL}$ extraction cell at the bottom of which a cellulose paper was inserted. The cell was filled up with some additional sand. Samples were automatically extracted by two consecutive cycles using a water-ethanol mixture $(1: 1 \mathrm{~V} / \mathrm{V})$ for $5 \mathrm{~min}$ at $40{ }^{\circ} \mathrm{C}$ and 1500 psi. The collected extracts (approximately $16 \mathrm{~mL}$ ) were made up to 20 $\mathrm{mL}$ with the extracting solvent mixture. After centrifugation for $5 \mathrm{~min}$ at $5000 \mathrm{~g}$ at room temperature, supernatants were filtered through a $0.2 \mu \mathrm{m}$ PTFE filter and aliquots $(50 \mu \mathrm{L})$ were diluted with water $(150$ $\mu \mathrm{L})$ and used for further LC-MS analyses.

\subsection{UPLC-TOF-HRMS}

UPLC analysis was performed with an Acquity ${ }^{\mathrm{TM}}$ instrument from Waters Corporation (Baden-Dättwil, Switzerland). For the separation, an Acquity UPLC ${ }^{\circledR} \mathrm{BEH}$ $\mathrm{C} 18(50 \times 2.1 \mathrm{~mm}, 1.7 \mu \mathrm{m})$ analytical column was used (Waters Corporation). The mobile phase consisted of water (phase A) and acetonitrile (phase B) with $0.1 \%$ formic acid. The gradient started with $2 \%$ of phase B, and was linearly increased to $20 \%$ in $2 \mathrm{~min}$ and then to $100 \%$ in $0.1 \mathrm{~min}$, kept constant for $0.9 \mathrm{~min}$ and then decreased in 0.1 min back to $2 \%$. Column re-equilibration time was set at $0.9 \mathrm{~min}$. The column temperature was maintained at $40{ }^{\circ} \mathrm{C}$, and 
the flow rate was set at $1 \mathrm{~mL} \mathrm{~min}^{-1}$. The injection volume was $1 \mu \mathrm{L}$.

Exact mass analysis of caffeine was performed with a Waters LCT Premier ${ }^{\mathrm{TM}}$ TOF-HRMS in positive ESI mode. Instrument calibration was achieved using formate and leucine-enkephalin solutions, which were infused through a LockSpray source during analysis. MS acquisitions with ion flight path in $\mathrm{W}$-mode were based on a dynamic screening, ranging between 180 and $1000 \mathrm{~m} / \mathrm{z}$ with a scan time of $0.25 \mathrm{~s}$ and a centroid data acquisition. The capillary, sample cone and aperture 1 voltages were set at 2400, 40 and $15 \mathrm{~V}$, respectively. The source and desolvation temperatures were set at 120 and $350{ }^{\circ} \mathrm{C}$, and cone and desolvation gas flows were set at 800 and $20 \mathrm{~L} \mathrm{~h}^{-1}$, respectively. A MassLynx V4.1 from Waters was used for data acquisition and processing.

\subsection{GC $\times G C-T O F-M S$}

Analyses were performed using a Pegasus $^{\circledR}$ 4D GC $\times$ GC-TOF-MS (Leco, Saint Joseph, MI, USA) equipped with a programmed temperature vaporised inlet coupled to a TDU and a MultiPurpose Sampler from Gerstel (Mülheim an der Ruhr, Germany). Powdered linden was directly analysed by thermal desorption by depositing $1-10 \mathrm{mg}$ of sample on the glass wool of a straight $4 \mathrm{~mm}$ i.d. desorption tube. The TDU temperature was programmed from 50 (held for $0.1 \mathrm{~min}$ ) to

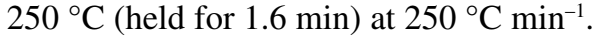
Desorption was operated in the split mode (split ratio 10:1) and the temperature of the transfer interface was fixed at $270{ }^{\circ} \mathrm{C}$. The temperature programme of the cooled injection system (CIS) started from $-100{ }^{\circ} \mathrm{C}$ (equilibration time of $0.5 \mathrm{~min}$ ) and rose to $270{ }^{\circ} \mathrm{C}$ (held for $15 \mathrm{~min}$ ) at $12{ }^{\circ} \mathrm{C} \mathrm{s}^{-1}$. Injection occurred in split mode (split ratio 10:1). The carrier gas was helium set at a flow rate of $1 \mathrm{~mL} \mathrm{~min}{ }^{-1}$. An Rtx-200 column $(20 \mathrm{~m} \times 0.18 \mathrm{~mm}$ i.d., $0.2 \mu \mathrm{m}$ trifluoropropylmethyl polysiloxane film thickness) from Restek (Lisses, France) was used in the first dimension and the oven temperature was programmed from 50 to $300{ }^{\circ} \mathrm{C}$, at $10{ }^{\circ} \mathrm{C} \mathrm{min}^{-1}$. Modulation period was set at $6 \mathrm{~s}$, with $0.6 \mathrm{~s}$ of hot pulse and $2.4 \mathrm{~s}$ of cool time between stages. The second dimension column was a VF-17MS $(2 \mathrm{~m} \times 0.15 \mathrm{~mm}$ i.d., $0.15 \mu \mathrm{m} 50 \%$ phenyl, $50 \%$ dimethylpolysiloxane film thickness) from Varian (Agilent Technologies, Plan-les-Ouates, Switzerland), which was placed in the secondary oven and heated with a temperature offset of $+10^{\circ} \mathrm{C}$ relative to the first dimension oven. The TOF-MS was operated at a rate of 100 spectra $\mathrm{s}^{-1}$ from $m / z 35$ to 500, in the electron-impact ionisation mode at $70 \mathrm{eV}$. Ion source temperature was set at $220^{\circ} \mathrm{C}$, and the detector voltage was set at $1450 \mathrm{~V}$.

\subsection{UPLC-MS/MS Conditions}

UPLC analysis was performed with the same instrument, column and parameters as those described for the UPLC-TOFHRMS analysis.

Identification and quantification were performed in the positive electrospray ionisation (ESI) mode with a 4000 Q-trap hybrid mass spectrometer from $\mathrm{AB}$ Sciex (Applied Biosystems, Darmstadt, Germany). MS acquisition was based on a conventional selected reaction monitoring (SRM) mode by targeting caffeine at two specific mass transitions, one for quantification and one for confirmation. MS parameters were set as follows: curtain gas at $40 \mathrm{psi}$, collision gas at $5 \mathrm{psi}$, ion spray voltage at $5000 \mathrm{~V}$, source temperature at $500{ }^{\circ} \mathrm{C}$, ion source gas 1 at $40 \mathrm{psi}$, ion source gas 2 at 50 psi and dwell times for each SRM were $150 \mathrm{~ms}$. Declustering and entrance potentials were fixed at 51 and $10 \mathrm{~V}$, respectively. Collision energies and collision cell exit potentials were set at 31 and $26 \mathrm{~V}$, respectively for the 195.1/138.0 Thomson (Th) transition, and at 33 and 8 $\mathrm{V}$, respectively for the confirmation mass transition 195.1/110.1 Th. Acquisition and data treatment were carried out with Analyst V1.5.1. software from AB Sciex.

\subsection{Calibration and Validation}

A stock solution was prepared by dissolving an accurately weighted amount of approximately $10 \mathrm{mg}$ of caffeine in $10 \mathrm{~mL}$ of water-ethanol mixture $(1: 1 \mathrm{~V} / \mathrm{V})$. This stock solution was further diluted to generate calibration curves ranging from 10 to $2000 \mathrm{ng} \mathrm{mL}^{-1}$.
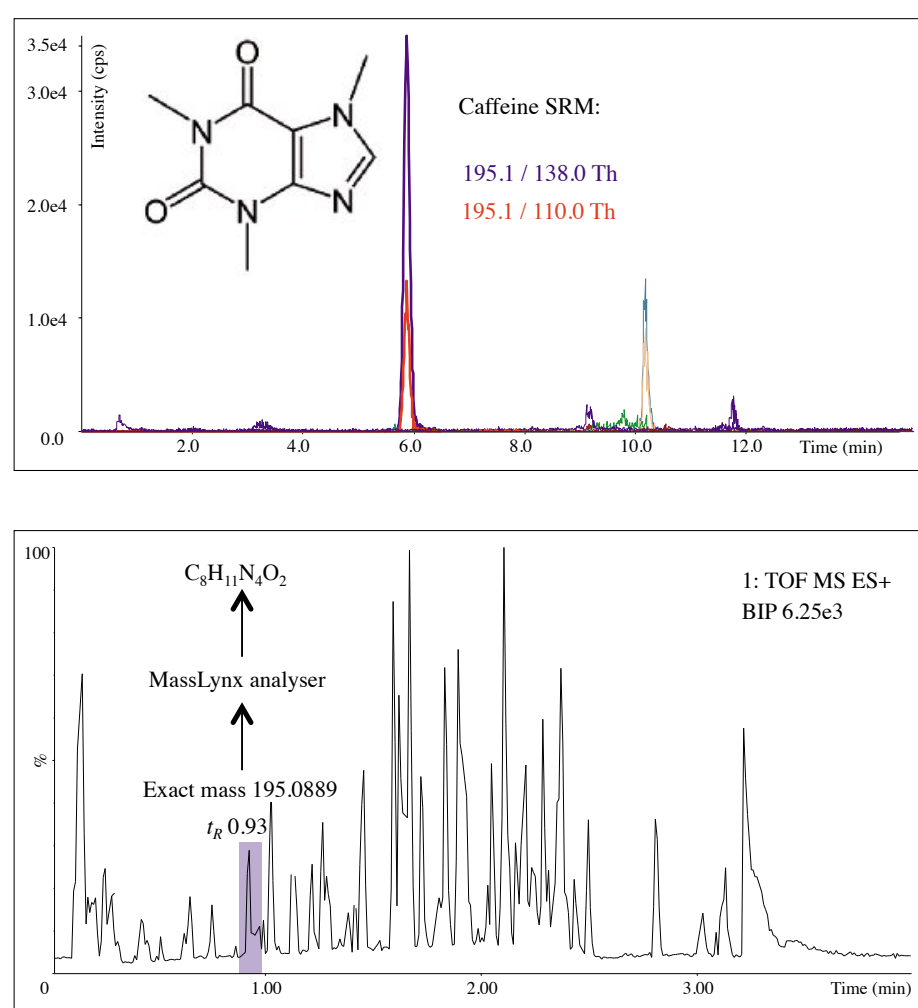

To validate both extraction processes and the analytical method, caffeine was spiked in linden extracts and also in linden teas. The spiked concentrations were between 100 and $1500 \mathrm{ng} \mathrm{mL}^{-1}(100,500$, 1000 and $\left.1500 \mathrm{ng} \mathrm{mL}^{-1}\right)$. The four validation standards (VS) were replicated three times and repeated in three independent series.

\subsection{Brewing Times}

To evaluate the extraction of caffeine in boiling water, linden tea was prepared and caffeine was quantified at increasing steeping times. For this, one bag of linden tea was brewed in boiled water $(200 \mathrm{~mL})$ and sampled after 1, 2, 5, 10, 20 and 30 min of slight agitation at room temperature. These experiments were performed in triplicate. Two $\mathrm{mL}$ was collected and prepared for UPLC-MS/MS analysis as described above in section 2.5.

\section{Results and Discussion}

During a survey of food supplements by HPLC-MS/MS, ,5] caffeine was detected in sleep-inducing foodstuff (Fig. 1). The presence of caffeine in the latter was surprising as caffeine acts as a weak psychostimulant and is known to cause trouble with sleeping. ${ }^{[4]}$ Investigations were conducted to exclude any possible misidentifications. Each botanical indicated on the product packaging of the sedative mixtures was analysed by UPLC-TOF-HRMS, and caffeine was detected in linden (Tilia spp.) extracts (Fig. 2). The measured exact mass in linden teas, as well as in linden pow-

Fig. 1. Caffeine structure and HPLC-MS/ MS chromatogram in selected reaction monitoring (SRM) mode of an extract of sleep-aid supplement.

Fig. 2. UPLC-TOFHRMS chromatogram of linden tea. The exact mass of 195.0889 Th corresponds to the protonated caffeine formula: $\mathrm{C}_{8} \mathrm{H}_{11} \mathrm{~N}_{4} \mathrm{O}_{2}$. 
der extracts, was 195.0889 Th, which has a difference of $0.7 \mathrm{mDa}$ with the theoretical exact mass of caffeine: $195.0882 \mathrm{Th}$. Retention times (0.93 min) in linden samples were identical to the caffeine standard and the isotope cluster matched very well with the predicted isotope peaks of caffeine (the normalised isotope fit (i-FIT) was 0.0 ). Thus UPLC-TOF-HRMS analyses confirmed the presence of caffeine in linden teas.

To corroborate these results and to exclude any contamination in linden teas or extracts, powdered lindens were directly analysed using a TDU coupled with a GC $\times$ GC-TOF-MS (Fig. 3). These direct desorption processes were conducted on caffeine standards and powdered lindens. Caffeine was chromatographically identified in all samples by the two retention times, $1140 \mathrm{~s}$ in the first GC dimension and $5.05 \mathrm{~s}$ in the second GC dimension, respectively (Fig. 3B). The mass spectrum of the molecular ion $194 \mathrm{Th}$, generated at $70 \mathrm{eV}$ (Fig. 3D), was compared with the NIST library, and the first hit was caffeine with at least a similarity of 795/1000 (Fig. 3C). As caffeine was unambiguously identified in various linden samples, an UPLC-MS/ MS method was developed and validated to measure the concentration levels in linden tea preparations (Fig. 4).

\subsection{Validation}

Based on protocol V5 of the SFSTP (Société française des sciences et techniques pharmaceutiques) proposal, the validation design consisted of three independent series of one blank and six calibration points, replicated three times, and with four VSs replicated three times. ${ }^{[14]}$ The spiked and non-spiked samples were analysed and the recoveries were calculated by comparing the added and the back-calculated concentrations of the VSs using external standard calibration curves. The response function was linear within the selected range ( 0.01 to $\left.2.0 \mathrm{mg} \mathrm{L}^{-1}\right)$. Backcalculated concentrations of the VSs were estimated by linear regression using different mathematical transformations (e.g. weighted factors: $1 / \mathrm{X}$ and $1 / \mathrm{X}^{2}$, squareroot transformation and log transformation) without applying a correction factor. Accuracy profiles were built to visualise the most appropriate regression (Figs 5 and 6). For ASE extracts, caffeine was quantified using linear regression without mathematical transformation (Fig. 5), while a $1 / \mathrm{X}$ weighted factor was applied for the quantification of linden tea (Fig. 6). Confidence intervals were between 11 and $20 \%$ for the four VSs of the ASE linden extraction process $(40,200,400$ and $600 \mathrm{mg}$ per $\mathrm{kg}$ of powdered linden) and between 17 and $20 \%$ for linden teas $(0.08,0.40,0.80$ and $1.20 \mathrm{mg} \mathrm{cup}^{-1}$ ).
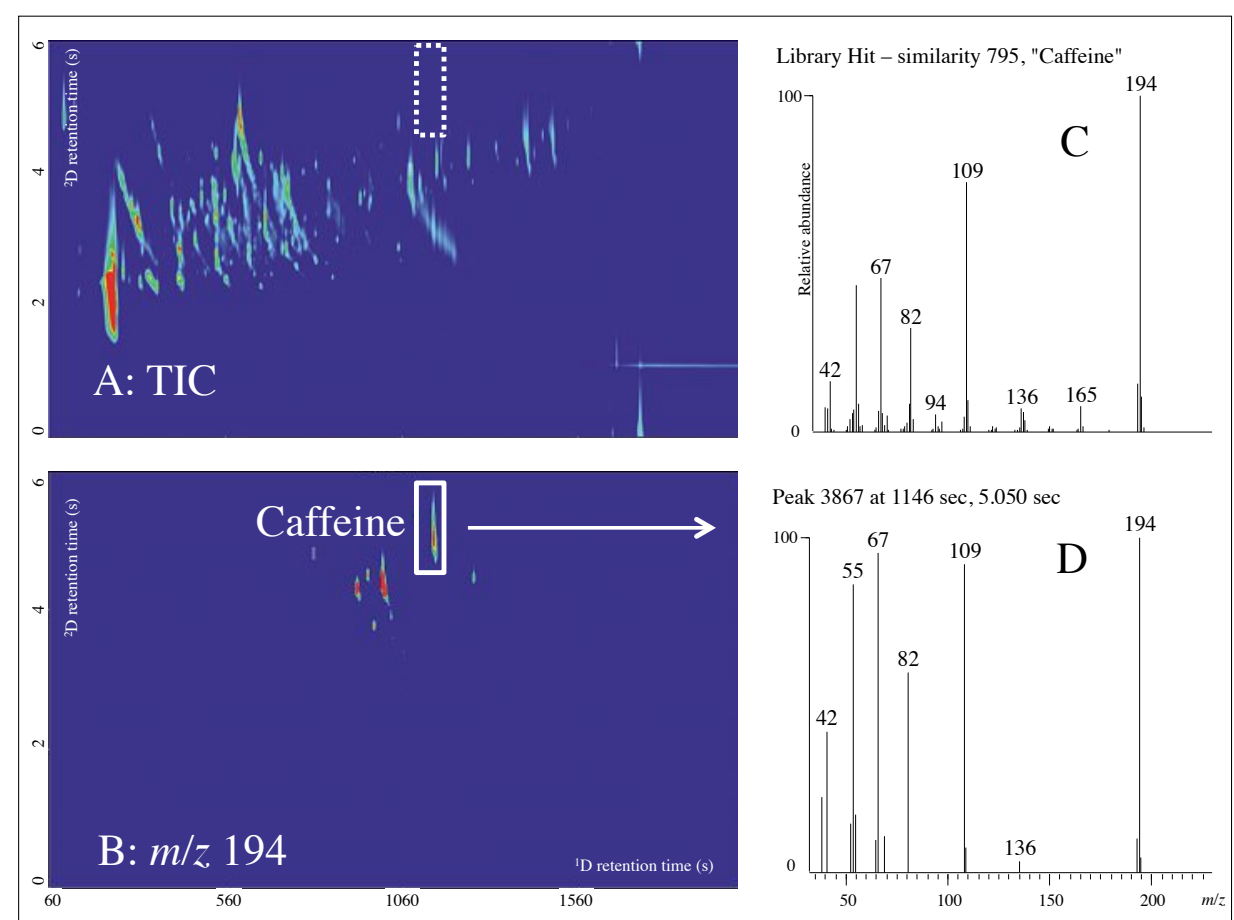

Fig. 3. GC $\times$ GC-TOF-MS data of a powdered linden sample extracted using a thermal desorption unit. A: total ion chromatogram (TIC). B: chromatogram of the selected $\mathrm{m} / \mathrm{z} 194$ ion. C: identification of caffeine based on MS/MS spectra comparison with the NIST library. D: MS/MS spectra of caffeine in linden tea.

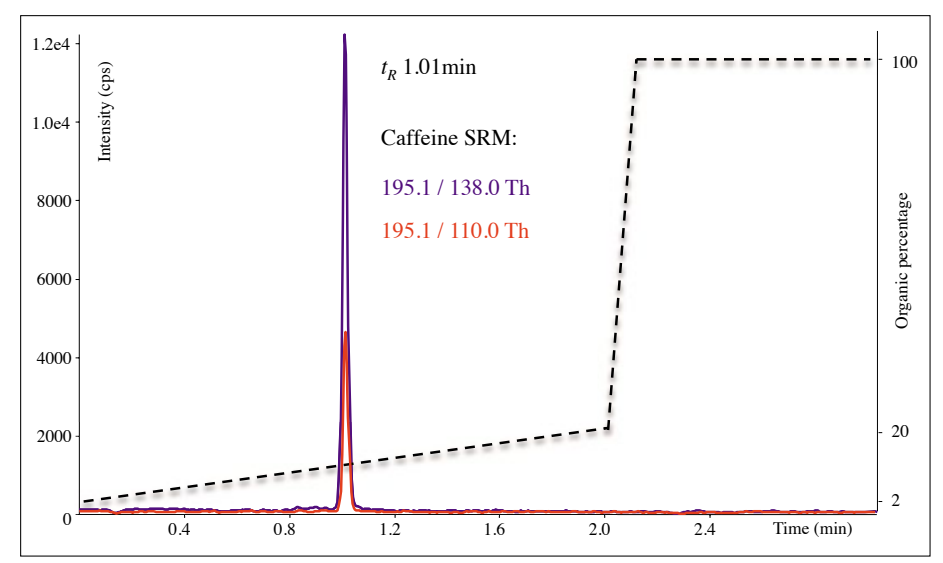

Fig. 4. UPLC-ESI-MS/ MS chromatogram in selected reaction monitoring (SRM) mode of a linden tea and the corresponding UPLC gradient.

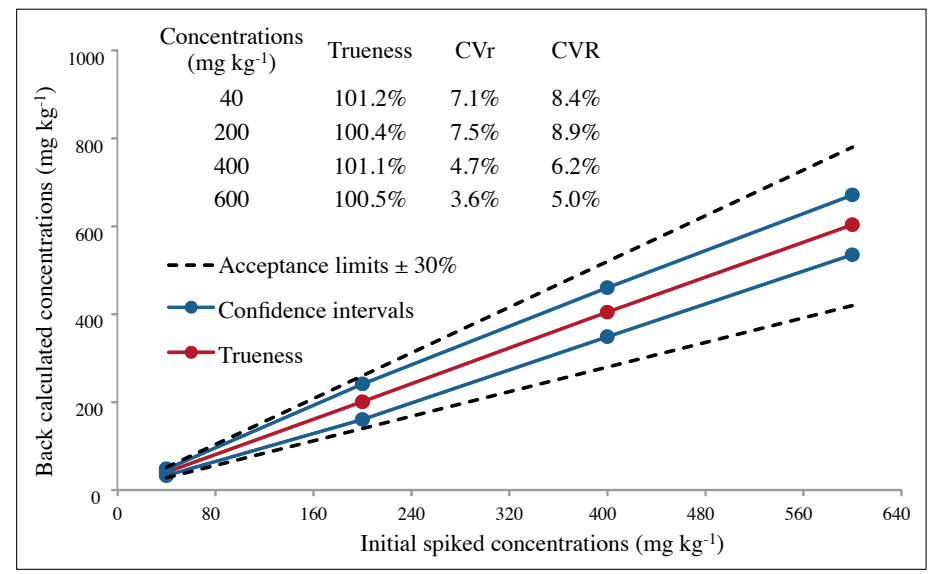

Fig. 5. Accuracy profile of ASE extracts in the concentration range from 40 to 600 $\mathrm{mg} \mathrm{kg}^{-1}$. Acceptance limits are set at $\pm 30 \%$. CVr: repeatability; CVR: intermediate fidelity.

\subsection{Brewing Times}

To evaluate the extraction kinetic, caffeine was quantified at increasing steeping times $(1,2,5,10,20$ and $30 \mathrm{~min})$. After 30 min, $0.112 \pm 0.012 \mathrm{mg}$ of caffeine was extracted in the $200 \mathrm{~mL}$ of water, and meas- ured concentrations at 10,20 and $30 \mathrm{~min}$ were statistically not different. During the first 5 min of brewing, $62 \%$ of the caffeine was already extracted, showing that its concentration increased rapidly within the first minutes (Fig. 7). 


\subsection{Analysis of Linden Teas}

The validated methods were used to quantify caffeine in 11 linden-based preparations both by ASE and brewing (Tables 1 and 2). Caffeine was detected in all samples. Amongst these samples, one was an instant linden tea, three samples were loose samples and seven were packed in teabags. The instant tea was a mixture of sugar and several botanicals. The majority of the samples $(n=8)$ contained only linden flowers, Tilia flos; one sample was a mixture of linden and Phillyrea flowers; another tea was a mixture of linden, chamomile, and orange leaves and flowers. In this particular case, the occurrence of caffeine could also be due to orange leaves and flowers, as several studies reported the presence of caffeine in this plant. ${ }^{[15,16]}$

Table 1. Caffeine concentrations measured in linden teas. Limit of quantification $=0.08 \mathrm{mg}$ cup $^{-1}$

\begin{tabular}{|l|l|}
\hline Type of sample & Caffeine $\left(\mathrm{mg} \mathrm{cup}^{-1}\right)$ \\
\hline teabag & $0.082 \pm 0.013$ \\
\hline teabag & $0.090 \pm 0.014$ \\
\hline teabag & $0.116 \pm 0.016$ \\
\hline teabag & $0.130 \pm 0.017$ \\
\hline teabag & $0.226 \pm 0.023$ \\
\hline teabag & $<0.08$ \\
\hline teabag & $<0.08$ \\
\hline loose sample & $<0.08$ \\
\hline loose sample & $<0.08$ \\
\hline loose sample & $<0.08$ \\
\hline instant tea & $<0.08$ \\
\hline
\end{tabular}

Table 2. Caffeine concentrations measured in linden ASE extracts. Limit of quantification = $40 \mathrm{mg} \mathrm{kg}^{-1}$

\begin{tabular}{|l|l|}
\hline Type of sample & Caffeine $\left(\mathrm{mg} \mathrm{kg}^{-1}\right)$ \\
\hline teabag & $56.5 \pm 9.4$ \\
\hline teabag & $68.7 \pm 10.6$ \\
\hline teabag & $88.1 \pm 10.5$ \\
\hline teabag & $91.6 \pm 12.2$ \\
\hline teabag & $101.5 \pm 12.2$ \\
\hline teabag & $109.1 \pm 12.6$ \\
\hline teabag & $109.8 \pm 13.1$ \\
\hline loose sample & $<40$ \\
\hline loose sample & $<40$ \\
\hline loose sample & $<40$ \\
\hline instant tea & $<40$ \\
\hline
\end{tabular}

In six linden tea preparations, concentrations were below the LOQ $(0.080 \mathrm{mg}$ per cup), and for the others, amounts of caffeine were between $0.082 \pm 0.013$ and

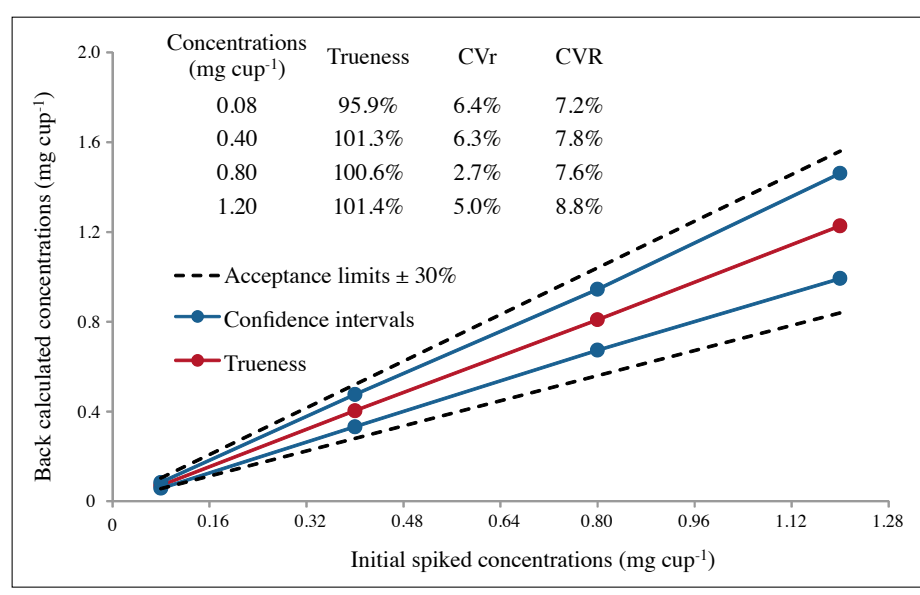

Fig. 6. Accuracy profile of linden teas in the concentration range from 0.08 to $1.20 \mathrm{mg} \mathrm{cup}^{-1}$. Acceptance limits are set at $\pm 30 \%$. CVr: repeatability; CVR: intermediate fidelity.

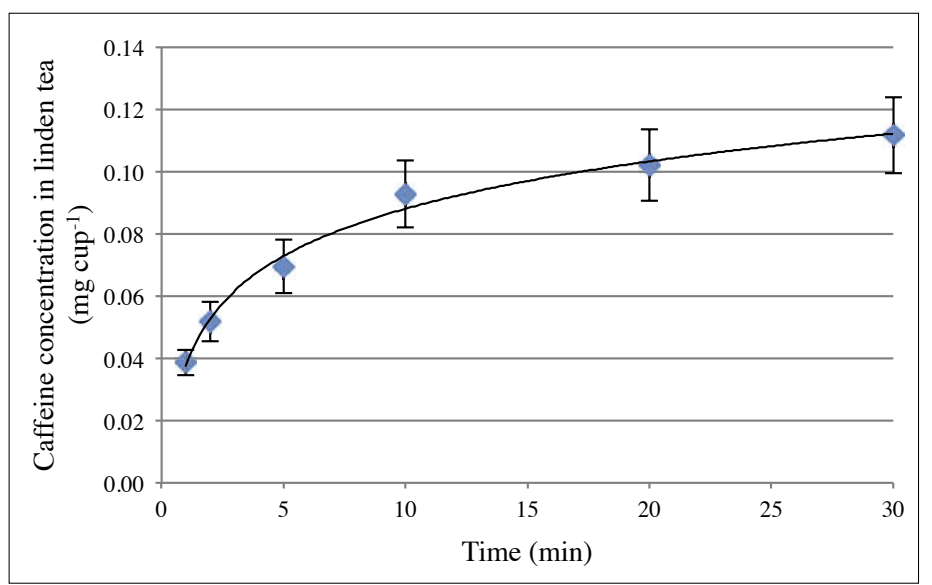

Fig. 7. Correlations between caffeine concentration and infusion time of linden tea.

$0.23 \pm 0.023 \mathrm{mg}$ per cup of tea (Table 1 ) For the ASE process (LOQ was $40 \mathrm{mg}$ $\mathrm{kg}^{-1}$ of linden powder), the concentrations measured ranged from $57 \pm 9$ to $110 \pm$ $13 \mathrm{mg} \mathrm{kg}^{-1}$ (Table 2).

\subsection{Significance of Caffeine Content}

Numerous studies have described the pharmacological effects of caffeine on humans, but in most of them, caffeine intakes were very large in comparison with the amounts of caffeine measured in classical tea or coffee. As far as we know, only one publication reports the physiological effect of small caffeine amounts after the consumption of coffees and decaffeinated coffees. ${ }^{[17]}$ In their study, Adan et al. stated that both coffees and decaffeinated coffees induced less somnolence and greater activation. The effect of decaffeinated coffee was greater in women than in men, but intakes were not quantified. Commonly observed caffeine concentrations in decaffeinated coffee are between 0 and $16 \mathrm{mg}$ per serving, ${ }^{[18]}$ and less than $12 \mathrm{mg}$ per serving in decaffeinated tea. ${ }^{[19]}$ In comparison, caffeine concentrations measured in conventional coffee ranged from 58 to 564 $\mathrm{mg}$ per serving, ${ }^{[20]}$ and from 14 to $61 \mathrm{mg}$ per serving in black, green and white teas, irrespective of the variety of tea.[19]

Concentrations observed in linden teas are largely lower than in coffees or teas and significantly lower than in decaffeinated hot beverages. While caffeine measured in linden tea has probably no physiological effects in adults, it may cause some disorders to small children. Indeed, Paterson and Wilson have reported that on the one hand, caffeine half-life is faster in adults ( 3.5 to $6 \mathrm{~h}$ ) than in children (ca. 4 days) where it may accumulate, and on the other hand, non-coffee drinkers are more sensitive to coffee's insomniac effect. ${ }^{[3]}$

\section{Conclusion}

In this study, caffeine was clearly identified in linden extracts and teas. Amounts are around ten times lower than in decaffeinated coffees or teas. Nevertheless, a consumption of several cups of such sleep-inducing preparations per day may cause some nervous disorders, especially in children. It would be interesting to evaluate the effect of the lowest observable concentration of caffeine on somnolence and stimulant activities.

\section{Acknowledgements}

We gratefully thank Yves Janin (Official Food and Veterinary Control Authority of Geneva) for assistance with GC×GC-TOF-MS analysis.

Received: July 3, 2014 
[1] J. Bruneton, 'Pharmacognosie: Phytochimie, Plantes Médicinales', Éditions Tec \& Doc, Paris, 2009.

[2] G. Fisone, A. Borgkvist, A. Usiello, Cell. Mol. Life Sci. 2004, 61, 857 .

[3] S. Bolton, G. Null, Orthomol. Psychiatry 1981, 10, 202.

[4] L. M. Paterson, S. J. Wilson, D. J. Nutt, P. H Hutson, M. Ivarsson, J. Psychopharmacol. 2009, 23, 475 .

[5] C. Mathon, M. Duret, M. Kohler, P. Edder, S Bieri, P. Christen, Food Chem. 2013, 138, 709.

[6] E. Aguirre-Hernandez, A.L. Martinez, M.E. Gonzalez-Trujano, J. Moreno, H. Vibrans, M. Soto-Hernandez, J. Ethnopharmacol. 2007. 109, 104.

[7] L. Rodriguez-Fragoso, J. Reyes-Esparza, S.W Burchiel, D. Herrera-Ruiz, E. Torres, Toxicol. Appl. Pharmacol. 2008, 227, 125.

[8] G. Toker, M. Aslan, E. Yesilada, M. Memisoglu, S. Ito, J. Pharm. Biomed. Anal. 2001, 26, 111.

[9] R. Naef, A. Jaquier, A. Velluz, B. Bachofen, Chem. Biodiversity 2004, 1, 1870.

[10] A.K. Atoui, A. Mansouri, G. Boskou, P. Kefalas, Food Chem. 2005, 89, 27.

[11] D. Horzic, D. Komes, A. Belscak, K.K. Ganic, D. Ivekovic, D. Karlovic, Food Chem. 2009, $115,441$.

[12] P. J. Eugster, J. L. Wolfender, 'UHPLC in Natural Products Analysis', in 'UHPLC in Life Sciences', The Royal Society of Chemistry, 2012, p. 354.

[13] X. Jun, J. Food Eng. 2009, 94, 105.

[14] P. Hubert, J. J. Nguyen-Huu, B. Boulanger, E. Chapuzet, P. Chiap, N. Cohen, P. A Compagnon, W. Dewé, M. Feinberg, M. Lallier, M. Laurentie, N. Mercier, G. Muzard, C. Nivet, L. Valat, E. Rozet, J. Pharm. Biomed. Anal. 2007, 45, 70 .

[15] E. Schievano, E. Morelato, C. Facchin, S. Mammi, J. Agric. Food Chem. 2013, 61, 1747.

[16] E. Schievano, M. Stocchero, E. Morelato, C. Facchin, S. Mammi, Metabolomics 2012, 8, 679.

[17] A. Adan, G. Prat, M. Fabbri, M. SanchezTuret, Prog. Neuro-Psychopharmacol. Biol. Psychiatry 2008, 32, 1698.

[18] R. R. McCusker, B. Fuehrlein, B. A. Goldberger, M. S. Gold, E. J. Cone, J. Anal. Toxicol. 2006 , 30, 611 .

[19] J. M. Chin, M. L. Merves, B. A. Goldberger, A. Sampson-Cone, E. J. Cone, J. Anal. Toxicol. 2008, 32, 702 .

[20] R. R. McCusker, B. A. Goldberger, E. J. Cone, J. Anal. Toxicol. 2003, 27, 520 . 